\title{
Fractura de cadera en el adulto mayor: epidemiología y costos de la atención
}

\author{
Hip fracture in the elderly: epidemiology and costs of care \\ Pech-Ciau BA, ${ }^{*}$ Lima-Martínez EA, ${ }^{*}$ Espinosa-Cruz GA, ${ }^{*}$ Pacho-Aguilar CR, ${ }^{*}$ \\ Huchim-Lara $\mathrm{O},{ }^{*}$ Alejos-Gómez RA
}

Escuela de Ciencias de la Salud. Universidad Marista de Mérida.

RESUMEN. Introducción: El objetivo general de la investigación fue describir la epidemiología y estimar los costos médicos directos de la fractura de cadera en el adulto mayor en México. Material y métodos: Se realizó un estudio observacional y transversal retrospectivo. Se utilizaron dos bases de datos obtenidas de la Dirección General de Información en Salud del período 2013-2018: egresos hospitalarios y procedimientos quirúrgicos. Las variables incluidas fueron: sexo, entidad federativa, edad, año de registro y tipo de fractura de acorde a la CIE-10; de igual forma, todos los insumos necesarios para la realización del procedimiento quirúrgico. Resultados: Se registraron 16,829 ingresos de pacientes con fractura de cadera. Las mujeres representaron $69 \%$ del total de pacientes, la edad en promedio fue de 79 años y la estancia hospitalaria fue de nueve días, 77\% de las fracturas fueron de cuello de fémur y el promedio de los costos médicos directos de los procedimientos ascendieron a USD \$45,122,228.00 para el período estudiado. Conclusión: El riesgo de caídas aumenta con la edad, especialmente en el grupo etario de 80-89 años, por lo que se espera que este tipo de patologías se incremente en los próximos años. De igual forma, los costos para la atención de estas fracturas representan un impacto económico para los sistemas de salud. De manera que la implementación de estrategias de prevención, por ejemplo, en caídas es el método más eficiente para contribuir al envejecimiento saludable.

Palabras clave: Cadera, fractura, anciano, costo, tratamiento.
ABSTRACT. Introduction: The aim of this study was to describe the epidemiology and to estimate the direct medical costs of hip fracture among elderly patients in Mexico. Material and methods: An observational, cross sectional and retrospective study was undertaken. Hospital discharge and surgical procedures for the period 20132018 were the databases used for the analysis, and obtained from General Directorate of Health Information. Variables included were sex, federal entity, age, year of discharge, and fracture type according to the CIE-10; and also, the supplies needed for the surgical procedures. Results: A total of 16,829 patients with hip fracture were discharge, $69 \%$ were women, and the mean for age was 79 years old and for the hospital stay length was nine days. The most frequent fracture type was the femur neck with $77 \%$ and the average medical costs was USD $\$ 45,122,228.00$. Conclusion: Falling risks increase with age, especially in patients among 80-89 years of age, hence, is expected that this type of pathology increases in the following years. The medical costs for treatment of hip fracture represents an economic impact on health services. For that reason, the implementation of prevention strategies, risk of falling for example, is the one of most efficient approach.

Keywords: Hip, fracture, elderly, cost, treatment.

\section{Nivel de evidencia: IV}

* Escuela de Ciencias de la Salud. Universidad Marista de Mérida. Mérida, Yucatán, México.

* Centro de Especialidades Médicas Las Américas. Mérida, Yucatán, México.

Correspondencia:

Esteban Alberto Lima-Martínez

Universidad Marista de Mérida, Periférico Norte, Tablaje catastral Núm. 13941Carretera Mérida-Progreso, C.P. 97300. Mérida, Yucatán, México

E-mail: estebanlimamtz@gmail.com

Recibido: 01-08-2020. Aceptado: 30-06-2021.

Citar como: Pech-Ciau BA, Lima-Martínez EA, Espinosa-Cruz GA, Pacho-Aguilar CR, Huchim-Lara O, Alejos-Gómez RA. Fractura de cadera en el adulto mayor: epidemiología y costos de la atención. Acta Ortop Mex. 2021; 35(4): 341-347. https://dx.doi.org/10.35366/103314 


\section{Introducción}

En 2017 la población de adultos mayores se incrementó más del doble que lo reportado en la década de $1980 .{ }^{1}$ Se estima que en 2020 dicha población supere al grupo de menores de cinco años y para 2050 sea de 2,000 millones de personas. ${ }^{2}$ Durante el proceso de envejecimiento ocurren cambios en las funciones corporales, tales como reducción de la masa muscular, de los rangos de movimiento y de alteraciones en la coordinación y el equilibrio., ${ }^{3,4}$ Las caídas son en parte resultado de dichos cambios en los adultos mayores, ya que conforme se incrementa la edad aumenta el riesgo de caídas, siendo las fracturas una de las consecuencias de estas últimas; de igual forma, la presencia de comorbilidades puede incrementar dicho riesgo. ${ }^{6,7,8}$

Se ha reportado mayor prevalencia de fractura de cadera secundaria a caídas en pacientes mayores de 65 años y es más frecuente en mujeres que en hombres. ${ }^{9}$ Después de los 50 años las mujeres caucásicas tienen casi el doble de riesgo de fractura de cadera que los hombres. ${ }^{10}$ Entre 20 y $40 \%$ de los adultos mayores con esta patología mueren dentro del primer año posterior a sufrir la lesión.., 11

En México la Secretaría de Salud registró 71,771 egresos hospitalarios por fractura de fémur entre 2002 y 2007, de los cuales cerca de la mitad ocurrieron en personas de 65 años o más. ${ }^{12}$ En la Ciudad de México la incidencia de fracturas de cadera asciende a 1,725 casos en mujeres y a 1,297 en hombres por cada 100,000 habitantes con una proyección de incremento de hasta de siete veces para el año $2050 .{ }^{13}$ En cuanto a costos, en el año 2002 el Instituto Mexicano del Seguro Social (IMSS) gastó cerca de \$18,307,184.00 MXN en la atención de fractura de cadera. ${ }^{14}$ En ese mismo año, el costo unitario de la atención de una fractura de cadera en 2002 se encontraba entre $\$ 55,128.50$ y $\$ 112,100.00 \mathrm{MXN}$ dependiendo del sistema mexicano de salud que atienda al paciente. ${ }^{11}$

Esta patología implica problemas que abarcan más que el daño ortopédico, impacta áreas como medicina interna, rehabilitación, siquiatría y economía de la atención sanitaria. ${ }^{15}$ Considerando el envejecimiento de la población, se ha estimado que mundialmente el número de fracturas de cadera aumentará a 2.6 millones para el 2025 y a 6.25 millones en 2050, implicando, por ende, un problema cada vez mayor de salud pública; ${ }^{16}$ el tratamiento de la patología en el adulto mayor dependerá del tipo de fractura, las más frecuentes son las fracturas intracapsulares y extracapsulares. Los tratamientos más frecuentes son osteosíntesis y artroplastía parcial o total..$^{17,18}$

Considerando lo anterior, el objetivo del presente estudio es describir la epidemiología y estimar los costos médicos directos de la fractura de cadera en el adulto mayor en México.

\section{Material y métodos}

Se realizó un estudio con un enfoque cuantitativo, diseño observacional y de corte transversal retrospectivo. ${ }^{19}$ Se consideraron todos los casos de fractura de cadera contenidos en los registros de egresos hospitalarios y de procedimientos quirúrgicos de la Dirección General de Información en Salud de la Secretaría de Salud en el período comprendido entre 2013 y 2018.

Las afecciones principales obtenidas de la base de datos y de acuerdo con la Clasificación Internacional de Enfermedades fueron: fractura del cuello del fémur (S720), fracturas pertrocanteriana (S721) y fractura subtrocanteriana (S722). Posteriormente, se filtró la base de acuerdo con el grupo de edad de interés, esto es, se incluyeron sólo los casos cuyo paciente fuese mayor de 60 años. ${ }^{2}$

Las variables analizadas en los casos de fractura de cadera registrados fueron: sexo, entidad federativa de registro, edad, año de registro y tipo de fractura. Se estimaron los costos médicos directos de los procedimientos quirúrgicos realizados a nivel nacional para fractura de cadera con información proporcionada por un hospital privado de la ciudad de Mérida, Yucatán en el mes de mayo de 2020 en pesos mexicanos. Los costos médicos directos incluyen todos aquéllos relacionados con los cuidados médicos, tales como el tipo de procedimiento quirúrgico, cuidados hospitalarios, honorarios médicos, insumos necesarios y estudios de gabinete. El costo de la intervención quirúrgica incluyó los honorarios del personal participante en el procedimiento (cirujano ortopedista, anestesiólogo, primer y segundo ayudante, enfermería). Para el servicio de fisioterapia y rehabilitación del paciente se consideró un promedio de 10 sesiones. La estancia intrahospitalaria se calculó con base en el costo por noche en una habitación estándar multiplicado por el número de días de estancia promedio en pacientes de la tercera edad, que fue de nueve días, ${ }^{20}$ así como los estudios de gabinete y fármacos empleados. Los servicios de enfermería se calcularon con base en los días de estancia promedio y la tarifa de cada turno. Los insumos de acuerdo con el tipo de intervención fueron: clavo centromedular con bloqueo cefálico, placa con tornillo deslizante, tornillos canulados, hemiartroplastía de Thompson, prótesis total de cadera cementada y prótesis total de cadera no cementada. Para cada tipo de intervención se empleó la siguiente ecuación:

Procedimiento quirúrgico $=[$ (honorarios médicos + insumos $)+($ costo estancia hospitalaria $*$ n días $)+($ honorarios de enfermería * turnos * días de estancia promedio) + (costo de sesión fisioterapia * n sesiones)] [número de procedimientos]

Los costos fueron posteriormente convertidos a dólares usando como tasa de cambio 22.3 pesos mexicanos por dólar. Es importante mencionar que se empleó para todos los años la misma tasa y costos del año 2020.

Para la estimación de los costos anuales y totales, los procedimientos se agruparon en cuatro categorías de acuerdo con el catálogo de procedimientos CIE-9-MC (Tabla 1), obtenidas de la base de datos de procedimientos de los registros de egresos hospitalarios de la Dirección General de Información en Salud (DGIS): 
Tabla 1: Registro de procedimientos para el tratamiento de fractura de cadera.

$\begin{array}{lrrrrrr} & & & 2018 \\ \text { Procedimiento } & 2013 & 2014 & 2015 & 2016 & 2017 & \\ & & & & & & \\ \text { Sustitución o reemplazo total de cadera } & 116 & 76 & 188 & 26 & 287 \\ \text { Sustitución parcial de cadera } & 246 & 332 & 216 & 65 & 166 & 158 \\ \text { Reducción abierta de fractura con fijación interna fémur } & 1,082 & 863 & 935 & 761 & 1,104 & 1,221 \\ \text { Reducción cerrada de fractura con fijación interna fémur } & 323 & 91 & 219 & 167 & 280 & 203 \\ \text { Total } & 1,767 & 1,362 & 1,558 & 1,019 & 1,693 & 1,869\end{array}$

Tabla 2: Costos estimados anuales y totales de los procedimientos para tratamiento de fracturas de cadera.

\begin{tabular}{|c|c|c|c|c|c|c|c|}
\hline Procedimientos & 2013 & 2014 & 2015 & 2016 & 2017 & 2018 & $\begin{array}{c}\text { Costo total en } \\
\text { dólares }\end{array}$ \\
\hline $\begin{array}{l}\text { STC DC, SPC, RAFI, } \\
\text { RCFI CCM }\end{array}$ & $8,852,329$ & $8,852,329$ & $7,828,831$ & $5,141,936$ & $8,559,005$ & $9,504,603$ & $45,979,153$ \\
\hline $\begin{array}{l}\text { STC DC, SPC, RAFI, } \\
\text { RCFI DHS }\end{array}$ & $8,359,782$ & $8,359,782$ & $7,494,876$ & $4,887,276$ & $8,132,030$ & $9,195,047$ & $44,042,666$ \\
\hline $\begin{array}{l}\text { STC NC, SPC, RAFI, } \\
\text { RCFI CCM }\end{array}$ & $8,883,539$ & $8,883,539$ & $7,879,414$ & $5,148,931$ & $8,597,481$ & $9,581,823$ & $46,201,790$ \\
\hline $\begin{array}{l}\text { STC NC, SPC, RAFI, } \\
\text { RCFI DHS }\end{array}$ & $8,390,993$ & $8,390,993$ & $7,545,459$ & $4,894,271$ & $8,170,506$ & $9,272,266$ & $44,265,303$ \\
\hline
\end{tabular}

1. 8,151 sustitución o reemplazo total de cadera.

2. 8,152 sustitución parcial de cadera.

3. 7,935 reducción abierta de fractura con fijación interna fémur.

4. 7,915 reducción cerrada de fractura con fijación interna fémur.

La sustitución o reemplazo total de cadera $(8,151)$ puede tratarse con dos técnicas diferentes: prótesis cementada o no cementada. ${ }^{21}$ Asimismo, para la reducción cerrada de fractura con fijación interna de fémur $(7,915)$ se consideraron dos técnicas distintas: osteosíntesis con clavo centromedular u osteosíntesis con tornillo deslizante (DHS). ${ }^{14,22}$ De éstas, dos categorías, se contemplaron ambas técnicas disponibles para cada una.

Para calcular el costo anual y total de los procedimientos se contemplaron cuatro escenarios, cada escenario incluía: costo de sustitución parcial de cadera ( $\$ 8,152$ dólares), costo de reducción abierta de fractura con fijación interna fémur ( $\$ 7,935$ dólares), costo de una de las dos técnicas disponibles para sustitución total de cadera $(\$ 8,151$ dólares) y costo de una de las dos técnicas de reducción cerrada con fijación interna fémur (\$7,915 dólares). Esto se hizo para cubrir todas las posibilidades de tratamiento ante la falta de especificidad en la base de datos empleada (Tabla 2).

Los análisis estadísticos se efectuaron con el software estadístico de versión libre Infostat ${ }^{\circledR}$. Se estructuró la descripción de la población estudiada mediante medidas de frecuencia y proporción para las variables categóricas, así como de tendencia central y dispersión para las numéricas.

\section{Resultados}

Se registró un total de 16,829 pacientes con fractura de cadera en el período entre 2013 y 2018. El número de casos en promedio por año fue de 2,804 \pm 388.98 (IC 95\%: 5.87). Del total, 11,689 fueron mujeres (69\%) y 5,140 hombres (31\%). En el período estudiado, el año con más casos reportados fue 2016 con 3,304, mientras que el año con menos casos fue 2013 con 2,271. Los casos por año se representan en la Figura 1. La edad promedio de los pacientes fue de 79 años \pm 9.26 (IC 95\%: 0.139). En cuanto a la distribución por grupos de edad, se observó en todos los años un mayor número de casos en el grupo etario de 80-89 años (Figura 2). En promedio, la entidad federativa con el menor número de casos fue Tabasco (125 casos), mientras que Guanajuato tuvo el mayor número de casos ( 3,100 casos) durante los últimos tres años del período estudiado, tal como se observa en la Figura 3. En los seis años estudiados la fractura de cuello del fémur fue la más frecuente con 12,986 casos, seguida de fractura pertrocantérica con 3,127 casos, mientras que la fractura subtrocantérica fue la menos frecuente con 716 casos (Figura 4). E1 promedio de estancia hospitalaria fue de nueve días.

La reducción abierta de fractura con fijación interna fémur fue el procedimiento realizado con mayor frecuencia durante el período 2013-2018, tal como puede observarse en la Tabla 1. 
La estimación de los costos anuales y totales tomando en cuenta las cuatro posibles combinaciones de procedimientos se muestran en la Tabla 2. El promedio de los costos médicos directos de los procedimientos para la fractura de cadera fue de $\$ 45,122,228.00$ dólares para el período 2013-2018. El costo unitario de cada intervención fue de $\$ 5,618.52$ dólares para osteosíntesis de cadera con clavo centromedular, $\$ 4,093,615$ dólares para osteosíntesis de cadera con tornillo deslizante (DHS), $\$ 4,990,057$ dólares para osteosíntesis de cadera con tornillos canulados, $\$ 3,954,848$ dólares para hemiartroplastía de cadera con prótesis tipo Thompson, $\$ 5,736,297$ dólares para artroplastía total de cadera cementada y $\$ 6,005,355$ dólares para artroplastía total de cadera no cementada.

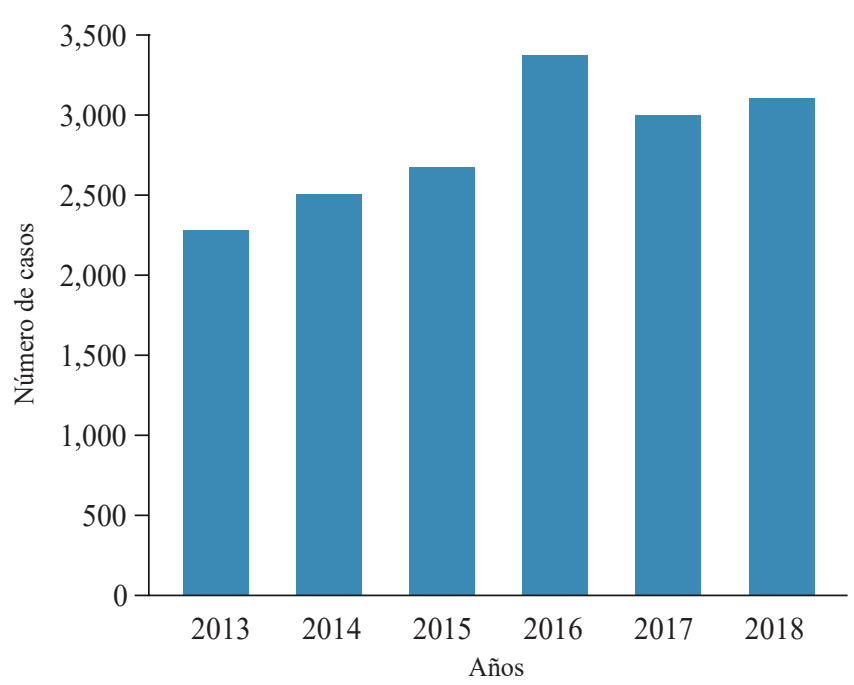

Figura 1: Número de casos por año.

\section{Discusión}

El componente descriptivo del estudio demuestra que el promedio de edad del paciente que sufre fractura de cadera fue de 79 años entre 2013 y 2018. Esto es relevante, ya que el riesgo de caídas aumenta exponencialmente con la edad; a partir de los 65 años $28 \%$ de los individuos sufre una caída y a los 80 años el riesgo será de $50 \%$. Esto aunado a que el grupo etario es vulnerable a diversos factores como déficit de la agudeza visual, osteoporosis, baja resistencia ósea, entre otros, que hacen a este grupo susceptible de sufrir una fractura de cadera. ${ }^{6,713,23}$ Este estudio revela que el grupo etario de 80-89 años es el más afectado por fracturas de cadera. Estos resultados son similares a los reportados por Cujilema, quien señala que la vulnerabilidad de este grupo está dada por mecanismos de trauma leve secundarios a una baja resistencia ósea por disminución de la densidad ósea en pacientes de edad avanzada. ${ }^{9}$

La frecuencia de las fracturas de cadera en mujeres puede ser consecuencia de factores tales como una pelvis ósea más ancha con tendencia a la coxa vara, menor actividad personal, presencia de osteoporosis temprana y un promedio de vida mayor que el de los hombres. ${ }^{24,25}$ Las fracturas de cuello femoral representaron $77.16 \%$ del total de fracturas de cadera, lo que concuerda con lo descrito en países como Inglaterra y EUA, donde las fracturas intracapsulares fueron ligeramente más frecuentes que los demás tipos de fractura. ${ }^{11,26}$ Para disminuir los días de estancia intrahospitalaria, el tratamiento de la fractura de cadera debe darse dentro de las primeras 24-48 horas para permitir la movilización temprana y evitar complicaciones que incrementen la mortalidad como dolor, infecciones de tracto urinario y neumonías, úlceras por presión y enfermedad trombótica venosa. ${ }^{9,27,28}$

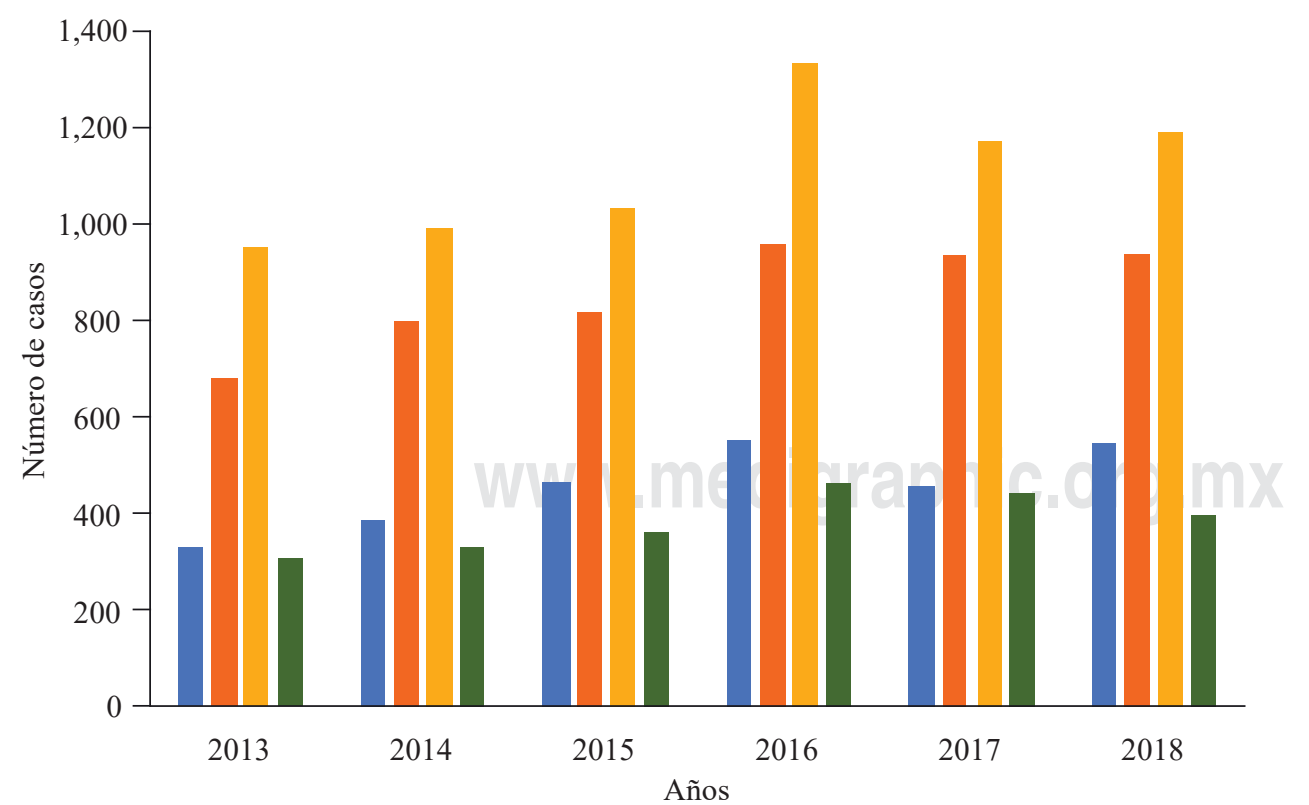

60-69

$70-79$

80-89

90 y más

Figura 2: 
En México la entidad federativa con más casos en el período estudiado fue Guanajuato; sin embargo, llama la atención que de 35 casos entre 2013 y 2015 se incrementan a 3,065 entre 2016 y 2018, lo anterior sugiere mayor investigación al respecto y/o una revisión de los reportes de vigilancia epidemiológica.

Considerando que el grupo etario afectado con más frecuencia por fractura de cadera encontrada en este estudio fue el de 80-89 años, es importante destacar el impacto económico que esto representa para los sistemas de salud y los pacientes. El envejecimiento de la población es un problema demográfico crucial y que se debe a la disminución de las tasas de nacimiento y mortalidad

Total de casos por entidad federativa en 2013

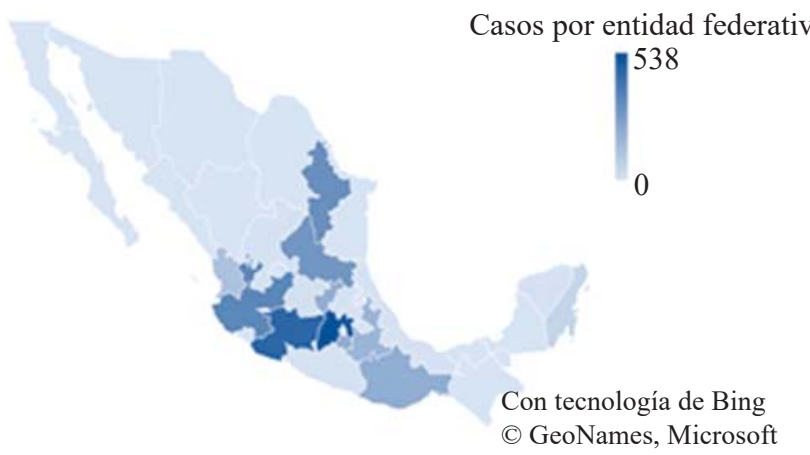

Total de casos por entidad federativa en 2015

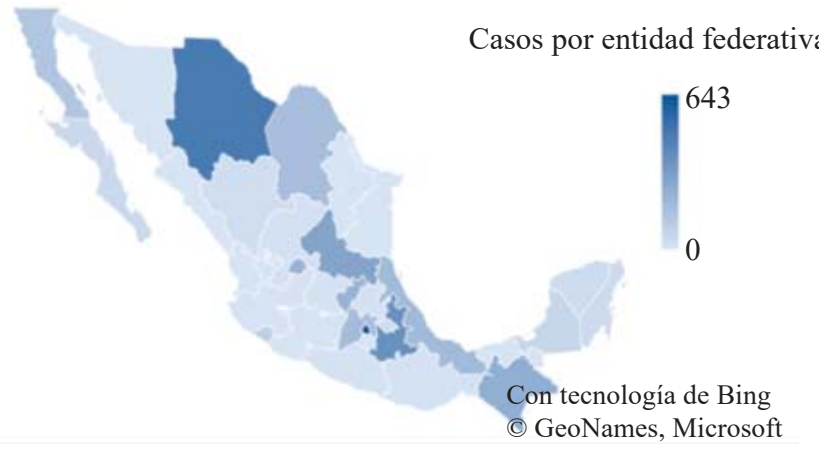

Total de casos por entidad federativa en 2017

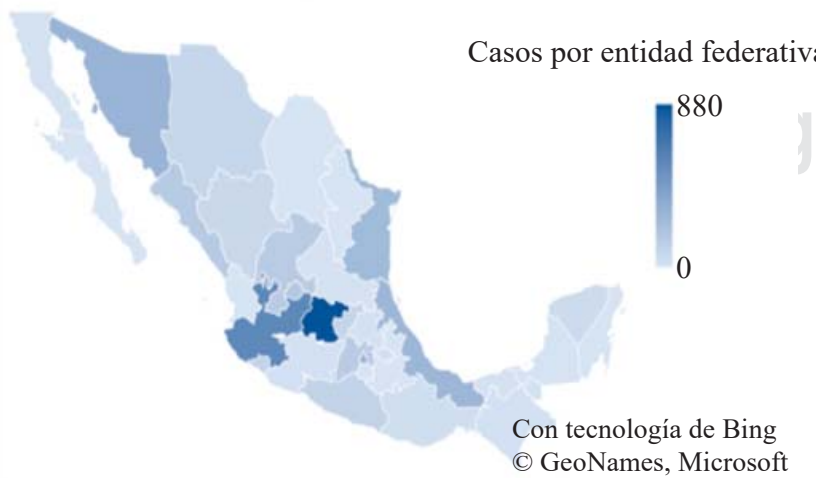

y a un incremento en la esperanza de vida. ${ }^{29}$ Se estima que la incidencia de fracturas de cadera se duplicará en 2050 en personas de 80 años en adelante y en ese mismo año $80 \%$ de la población de ancianos estará viviendo en países de bajo y mediano ingreso, por lo que las fracturas de cadera representarán un importante problema para todos los sistemas de salud. ${ }^{2,30}$

El número de procedimientos realizados fue en promedio de 1,544 al año, la reducción abierta con fijación interna de fémur se efectuó en más de $50 \%$, lo cual es congruente con la literatura, ya que para los pacientes en quienes la artroplastía no es la primera opción de tratamiento, la fijación interna con la colocación de tres tornillos

Total de casos por entidad federativa en 2014

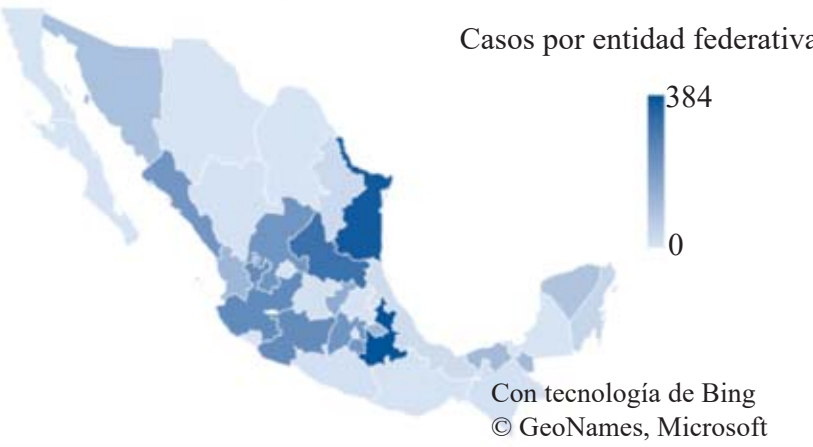

Total de casos por entidad federativa en 2016

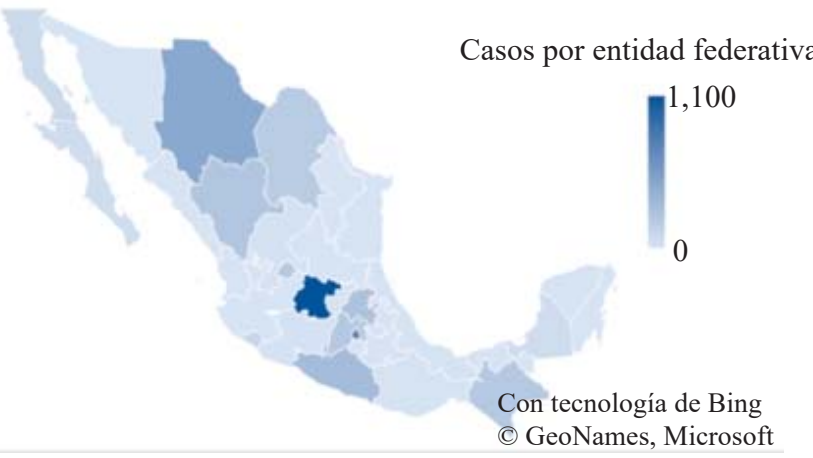

Total de casos por entidad federativa en 2018

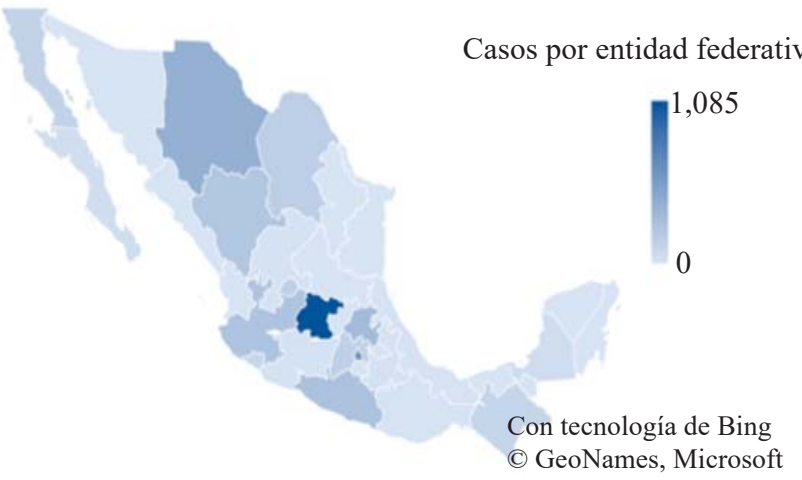

Figura 3: Distribución de casos por entidades. 
Fractura del cuello de fémur s720

Fractura pertrocantérica s721

Fractura subtrocantérica s722

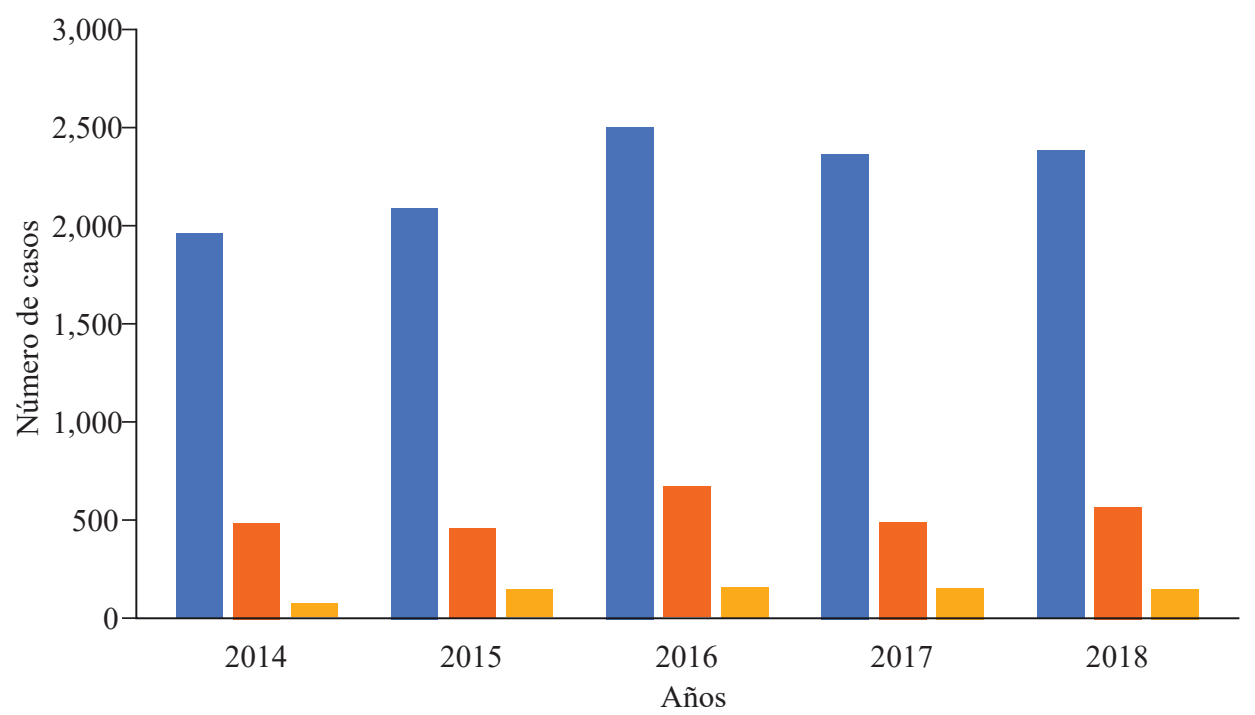

Figura 4: Casos por tipos de fractura.

canulados en configuración de triángulo invertido es una opción de tratamiento para las fracturas de cuello femoral; éstas representaron $77.16 \%$ de las fracturas totales en el estudio. ${ }^{31}$ No tenemos la evolución de estos casos, lo que sería importante para definir una mejor estrategia terapéutica al respecto.

El costo promedio anual de la atención a la fractura de cadera estimado entre 2013 y 2018 fue de \$7,950,293.54 dólares considerando precios de atención en un medio privado aislado y con una tasa de cambio fija, a pesar de ello, representa cuatro veces lo que el Instituto Mexicano del Seguro Social (IMSS) gastó en 2002. ${ }^{11}$ De manera general se recomienda realizar la artroplastía total en el adulto mayor de 65 años, el cual representa un gasto de entre $\$ 5,736.00$ y $\$ 6,005.00$ dólares, dependiendo del tipo de técnica. ${ }^{32}$ Este costo de salud tiene importancia, ya que se estima que es mayor que el costo de enfermedades cardíacas y cáncer de mama y ginecológicos. ${ }^{12.33}$

\section{Conclusión}

Las fracturas de cadera en el adulto mayor son frecuentes e impactan gravemente la calidad de vida de los pacientes afectados. Debido a que estas fracturas no sólo significan un costo extra para los sistemas de salud, sino que también tienen una alta tasa de mortalidad y complicaciones, la implementación de mejores y eficientes estrategias de prevención de caídas es el método más eficiente para contribuir al envejecimiento saludable en todo el país.

Referencias

1. United Nations Organization (ONU). World Population Ageing. 2017, 9-33.

2. World Health Organization (OMS). Ageing and Health. 2018.

3. Baptista H, Mendes D, Soares C. Age-related mobility loss is jointspecific: an analysis from 6,000 Flexitest results. Age (Dordr). 2013; 35(6): 2399-407.
4. Soucie J, Wang C, Forsyth A. Range of motion measurements: reference values and a database for comparison studies. Haemophilia. 2011; 17(3): 500-7.

5. Tinetti ME, Williams CS, Gill TM. Health, functional, and psychological outcomes among older persons with chronic dizziness. J Am Geriatr Soc. 2000; 48(4): 417-21.

6. Inouye S, Studenski S, Tinetti M, Kuchel G. Geriatric syndromes: clinical, research, and policy implications of a core geriatric concept. J Am Geriatr Soc. 2007; 55: 780-91.

7. Inouye $\mathrm{S}$, Brown $\mathrm{C}$, Tinetti $\mathrm{M}$. Medicare nonpaymenthospital falls unintended consequences. $N$ Engl J Med. 2009; 360: 2390-3.

8. Hernández J, Paz A, Huchim-Lara O, Méndez-Domínguez N. Riesgo de caídas y de sensibilidad periférica entre adultos mayores con diabetes. Fisioterapia. 2018; 40(5): 226-31.

9. Cujilema J, Palacio J, Stangl W. Resultados funcionales de hemiartroplastía bipolar en pacientes mayores de 65 años con fracturas intracapsulares de cadera. Acta Ortop Mex. 2019; 33(4): 241-6.

10. Koval J, López D. Fracturas femorales en el anciano. Editorial SECOT, Madrid. 2013.

11. Viveros JC. Fractura de cadera por fragilidad en México: ¿En dónde estamos hoy? ¿Hacia dónde queremos ir? Acta Ortop Mex. 2018; 32(6): 334-41.

12. Veronese N, Maggi S. Epidemiology and social costs of hip fracture. Injury. 2018; 49(8): 1458-60.

13. Valles JF, Malacara M, Mont Landerreche GG, Suárez CE, Cárdenas JL. Tratamiento quirúrgico de las fracturas de cadera. Acta Ortop Mex. 2010; 24(4): 242-7.

14. Rodríguez V, Enríquez G, Escalante O. Osteosíntesis de cadera con clavo centromedular: posición en decúbito lateral, sin mesa de fracturas. Acta Ortop Mex. 2016; 30(6): 279-83.

15. Rueda G, Tovar JL, Hernández S, Quintero D, Beltrán CA. Características de las fracturas de fémur proximal. Repert Med Cir. 2017; 26(4): 213-8.

16. Guzon O, Perez E, Crespí N. Mortality after osteoporotic hip fracture: incidence, trends, and associated factors. J Orthop Surg Res. 2019; 14(1): 203.

17. Florschutz AV, Langford JR, Haidukewych GJ, Koval KJ. Femoral neck fractures: current management. J Orthop Trauma. 2015; 29(3): 121-9.

18. Bhandari M, Swiontkowski M. Management of acute hip fracture. $N$ Engl J Med. 2017; 377(21): 2053-62.

19. Jacobsen K. Introduction to health research methods. Jones \& Bartlett Publishers; 2017.

20. Barrios A, Contreras E. Frecuencia de complicaciones en pacientes mayores de 60 años con fractura de cadera. Acta Ortop Mex. 2018; 32(2): $65-9$. 
21. Duque J, Navarro R, Ruiz J. Biomecánica de la prótesis total de cadera: cementadas y no cementadas. Canarias Médica y Quirúrgica. 2011; 25(9): 32-46.

22. Opazo A. Fractura de cadera en el adulto mayor: manejo y tratamiento. Medwave. 2011; 11(2): 1-4.

23. Negrete-Corona J, Alvarado-Soriano JC, Reyes-Santiago LA. Fractura de cadera como factor de riesgo en la mortalidad en pacientes mayores de 65 años. Acta Ortop Mex. 2014; 28(6): 352-62.

24. Muñoz G, Lavanderos F, Vilches A, Delgado M, Cárcamo H, Passalaqua H, Guarda M. Fractura de cadera. Cuad Cir. 2018; 22(1): 73-81.

25. Collazo Á, Boada S. Morbimortalidad por fractura de cadera. Rev Cubana Ortop Traumatol. 2000; 14(1-2): 21-5.

26. Inacio MC, Weiss JM, Miric A, Hunt JJ, Zohman GL, Paxton EW. A community-based hip fracture registry: population, methods, and outcomes. Perm J. 2015; 19(3): 29-36.

27. Clark P, Carlos F, Barrera C, et al. Direct costs of osteoporosis and hip fracture: an analysis for the Mexican healthcare system. Osteoporos Int. 2008; 19(3): 269-76.
28. Smektala R, Endres HG, Dasch B, et al. The effect of time to surgery on outcome in elderly patients with proximal femoral fractures. BMC Musculoskelet Disord. 2008; 9: 171.

29. Granados V, Sánchez S, Ramírez R. Costos por hospitalización de adultos mayores en un hospital general regional del IMSS. Rev Med Inst Mex Seguro Soc. 2018; 56(S 1): 64-70.

30. Bergstrom U, Jonsson H, Gustafson Y, Pettersson U, Stenlund H, Svensson $\mathrm{O}$. The hip fracture incidence curve is shifting to the right. Acta Orthop. 2009; 80(5): 520-4.

31. Guo J, Dong W, Yin B. Intramedullary nails with cannulated screw fixation for the treatment of unstable femoral neck fractures. J Int Med Res. 2019; 47(2): 557-68.

32. Ogino D, Kawaji H, Konttinen L. Total hip replacement in patients eighty years of age and older. J Bone Joint Surg Am. 2008; 90(9): 1884-90.

33. Piscitelli P, Iolascon G, Argentiero A. Incidence and costs of hip fractures vs strokes and acute myocardial infarction in Italy: comparative analysis based on national hospitalization records. Clin Interv Aging. 2012; 7: 575-83. 